\title{
Regression-contingent analyses: A reply to Altmann
}

\author{
KEITH RAYNER and SARA C. SERENO \\ University of Massachusetts, Amherst, Massachusetts
}

\begin{abstract}
We discuss three points from Altmann's (this issue) reply to our comment on the use of the regression contingent analysis. First, we again argue that the Altmann, Garnham, and Dennis (1992) paper leaves the impression that eye movements are a necessary index of readers being garden pathed. Second, we acknowledge that differences among previously published studies dealing with parsing strategies are as likely to be due to structural differences in the stimuli as they are to memory span differences or different reading strategies. Third, we argue that researchers should examine a large number of possible dependent measures that are obtainable from the eye movement record.
\end{abstract}

Altmann (1994) has responded to our comment (Rayner \& Sereno, 1994) on the use of the regression-contingent analysis introduced by Altmann, Garnham, and Dennis (1992) by making a number of points. Among his points are the following: (1) Syntactic garden paths do not necessarily result in regressive eye movements. (2) The differences between Rayner, Garrod, and Perfetti's (1992) and Altmann et al.'s (1992) studies are due to structural differences in the stimuli. (3) The regression contingent analysis is generalizable and helps alleviate certain artifacts inherent in using milliseconds per character as a measure of processing time. Altmann makes some other points with which we either agree or for which we have decided that no reply is necessary.

Point 1. Altmann (1994) provides two quotes which indicate that he and his colleagues do not equate regressions and garden paths. Our response is that providing these quotes is unnecessary, since we acknowledged that they were aware that they should not be equated. Altmann also notes a number of times that we incorrectly ascribed to him and his colleagues the assumption that the two are equated. What we said was that "they seem to be assuming that syntactic garden paths must result in regressive eye movements." This quote is then followed by a footnote explicitly noting that Altmann et al. (1992) acknowledge that regressions and garden paths are not to be equated, but that we believe their article conveys that impression to the reader. Consider, for example, the following quotes from Altmann et al.'s (1992) paper: "the regression-contingent measure enabled us to locate all garden path effects in only a small proportion of trials" (p. 685); "the discrepancy between the reading time and regression data suggests that the regression data reflect

Correspondence should be addressed to K. Rayner, Department of Psychology, University of Massachusetts, Amherst, MA 01003.

-Accepted by previous editor, Margaret Jean Intons-Peterson a minority of occasions on which subjects garden path" (p. 694); "reading times on trials in which a regression does occur (that is, reading times prior to a regression) may reflect the processing complexities of a garden path" (p. 696). Since Altmann et al. (1992) make the point in the first quote that the small percentage of trials refers to cases in which readers regress, we think it likely that many readers might infer that Altmann et al. (1992) equate the two. When one combines quotes such as these with the quote that we previously provided in our original comment (that noncontingent first-pass reading times can hide the disambiguating effects of certain variables), it seems even more likely that naive readers could conclude from Altmann et al.'s (1992) article that garden paths are equated with regressions.

Obviously, it would be fruitless for us or for Altmann to continue to provide quotes out of context. Our point was and always has been that Altmann et al.'s (1992) paper leaves the impression that regressions are a necessary index of readers' being garden pathed. We invite interested readers to read Altmann et al.'s (1992) paper and draw their own conclusions. The nature of the current exchange between us and Altmann has clarified the issues and should leave no ambiguity: the regression-contingent analysis is a potentially important tool to use, but regressions are not to be equated with garden paths.

Point 2. In his reply, Altmann (1994) suggests that the reasons for the disparities between Rayner et al.'s (1992) and Altmann et al.'s (1992) papers derive from structural differences in the target sentences (or in the context in which the sentences occur) and not from different reading strategies employed in the two studies or memory span differences between readers. A careful reading of our reply to Altmann et al. (1992) should make it clear that we agree that structural differences may well be the cause of the different pattern of results. Indeed, the thrust of the final conclusions in both Britt, Perfetti, Garrod, and Rayner's (1992) and Rayner et al.'s (1992) papers is that the extent to which context can influence initial parsing 
decisions may depend critically on the structural characteristics of the sentence types used in a given study. Altmann also suggests that it would have been more appropriate to reexamine Britt et al.'s data than to reexamine Rayner et al.'s (1992) data (see his note 4). There is a simple reason why we did not do so: there were too few regressions in Britt et al.'s study to justify a regressioncontingent analysis. Altmann (1994) himself uses the same logic with regard to data from Altmann, Garnham, and Henstra (1994).

To summarize, we are content with the notion that disparities among various published studies dealing with parsing strategies are as likely to be due to structural differences in stimuli as they are to different reading strategies or to memory span differences. Presently, the reasons for the differences remain uncertain, as we suggested in our original comment.

Point 3. Altmann (1994) cleverly attempts to turn the tables on us a bit by suggesting that our finding that the garden path effect in Rayner et al.'s (1992) study got stronger when regressions were eliminated is consistent with data reported by Altmann et al. (1992). In particular, he notes that in the null context conditions of Altmann et al.'s (1992) studies when trials including regressions were eliminated from the data analysis, the garden path effect was stronger. He thus equates his null context conditions with the biasing context conditions used by Rayner et al. (1992) on the basis that the latter's contexts contained just one referent for the head of the noun phrase. Altmann (1994) has argued that such contexts need to be contrasted with others containing more than one referent in order best to assess contextual influences on initial parsing strategies. ${ }^{1}$

This issue was addressed in Rayner et al.'s (1992) article, and here we simply repeat that it is not obvious that Altmann (1994) is correct in his assertion that more than one referent is needed in the context (cf., e.g., Clifton \& Ferreira, 1989). The more critical issue is whether the regression-contingent analysis can be a useful measure to adopt in conjunction with analyses of first-pass reading times. Here, we largely agree with Altmann's (1994) arguments concerning potential problems with the first-pass reading time measure. Indeed, as he notes, some of the points were originally made by us (Rayner, Sereno, Morris, Schmauder, \& Clifton, 1989) and some are based in part on comments that we made in our review of his reply. However, some of the most important points are original to him. For some time now, we (see Rayner et al., 1989) have argued that researchers should examine a large number of possible dependent measures that are obtainable from the eye movement record. We also agree with the final two sentences of Altmann's (1994) reply to us with regard to eye movement studies. But we obviously think that examination of the eye movement record is perhaps the best way to investigate on-line processes in reading.

In summary, we hope that the exchange between Altmann and ourselves clarifies our positions. It seems that we agree that the regression-contingent analysis is a tool that can be used to accumulate additional data when one is examining initial parsing strategies. It may also be useful in the examination of issues about reading comprehension in general. But, in the case of parsing, its use should not be seen as being consistent with the notion that regressions and garden paths go hand in hand. It should now be clear that we and Altmann are in agreement on this point.

\section{REFERENCES}

AltmanN, G. T. M. (1994). Regression-contingent analyses of eye movements during sentence processing: Reply to Rayner and Sereno. Memory \& Cognition, 22, 286-290.

Altmann, G. T. M., Garnham, A., \& Dennis, Y. (1992). Avoiding the garden path: Eye movements in context. Journal of Memory \& Language, 31, 685-712.

Altmann, G. T. M., Garnham, A., \& Henstra, J. A. (1994). Effects of syntax in human sentence parsing: Evidence against a structurebased proposal mechanism. Journal of Experimental Psychology: Learning, Memory, \& Cognition, 20, 209-216.

Britt, M. A., Perfetti, C. A., Garrod, S., \& Rayner, K. (1992), Parsing in discourse: Context effects and their limits. Journal of Memory \& Language, 31, 293-314.

Clifton, C., \& Ferreira, F. (1989). Ambiguity in context. Language \& Cognitive Processes, 4, SI77-104.

Rayner, K., Garrod, S., \& Perfetti, C. A. (1992). Discourse influences during parsing are delayed. Cognition, 45, 109-139.

Rayner, K., \& Sereno, S. C. (1994). Regressive eye movements and sentence parsing: On the use of regression-contingent analyses. $\mathrm{Mem}$ ory \& Cognition, 22, 281-285.

Rayner, K., Sereno, S. C., Morris, R. K., Schmauder, A. R., Clifton, C. (1989). Eye movements and on-line language comprehension processes. Language \& Cognitive Processes, 4, SL21-50.

\section{NOTE}

1. Altmann et al. (1992) did provide two referents in both their complement-supporting contexts (e.g., a man and a woman) and their relative-supporting contexts (e.g., two women). (See Table 1 in Altmann et al., 1992, for an example set of stimuli.) In both cases, one of the referents is typically mentioned as the direct object of a verb that is later used in the complement or relative clause of the target sentence. However, in the target sentences, only the relative target sentence (nonminimal attachment) preserves the relationship between the referent and the old (given) information. In the complement target sentence (minimal attachment), the relationship between the referent and the old information is disrupted in that a new entity becomes the object of the verb of the complement clause. Thus, it is not clear whether the differences between Rayner et al.'s (1992) and Altmann et al.'s (1992) studies are due to the number of referents or to other properties of the materials.

(Manuscript received July 2, 1993; revision accepted for publication July 30, 1993.) 